\title{
LOS PROBLEMAS DE CONOCIMIENTO UNA PERSPECTIVA COMPLEJA PARA LA ENSEÑANZA DE LAS CIENCIAS
}

\author{
Juan Carlos Orozco Cruz, Steiner Valencia Vargas, Olga Méndez Núñez, Gladys Jiménez Gómez y José Pablo \\ Garzón Ortiz. Profesores Departamento de Física - Universidad Pedagógica Nacional \\ Miembros Grupo Física y Cultura
}

La noción de conocimiento nos parece Una y evidente. Pero, en el momento en el que se la interroga, estalla, se diversifica, se multiplica en nociones innumerables, planteando cada una de ellas una nueva interrogación.

Edgar Morin El Método

\begin{abstract}
Some theoretical aims are made by Ecoperspectivas Research Group in order to development a set of criteria and proposals for science teaching in basic education. The idea of science like culture activity than makes possible the construction of different representations of social and natural world is in the center of the group considerations; in addition are introduced the conceptions of science teaching practices as emergency of subjectivities and the knowledge problems as a didactic criterion to describe sciences classroom processes.
\end{abstract}

\section{RESUMEN}

En este escrito se presentan algunos referentes conceptuales desde los cuales el Grupo Eco-Perspectivas del Departamento de Física de la Universidad Pedagógica Nacional, ha venido orientando la formulación, diseño e implementación de propuestas de enseñanza de las ciencias para la Educación Básica. Estos planteamientos vinculan concepciones desde las cuales se concibe la ciencia como una actividad de construcción de representaciones del mundo natural y social, las prácticas de su enseñanza como la emergencia de nuevas subjetividades y los problemas de conocimiento como una categoría alternativa para dar cuenta de su complejo devenir.

\section{PRESENTACIÓN}

Actualmente se hace relevante asumir el conocimiento como una actividad de la cultura y la ciencia como una actividad de construcción de explicaciones. Es decir, como un tipo de actividad que está social e históricamente legitimada y que como tal es contextual y provisional. En los contextos escolares es cada vez más sentida la necesidad de diseñar propuestas de innovación e investigación, que pongan en juego alternativas didácticas y metodológicas, que permitan a los estudiantes vivenciar experiencias de construcción de conocimiento y a los maestros comprender los procesos pedagógicos implicados en dicha construcción.

Como una estrategia para responder a estas demandas el Grupo de Investigación EcoPerspectivas, del Departamento de Física de la Universidad Pedagógica nacional, ha configurado una manera de entender las prácticas de enseñanza de las ciencias que vincula este carácter cultural de la actividad científica. Los referentes que se esbozan a continuación expresan este vínculo y han sido el producto del trabajo de revisión y discusión teórica y 
resultado de un ejercicio de implementación y sistematización de propuestas de aula en instituciones educativas del Distrito Capital.

Dicho ejercicio permite concebir la enseñanza de las ciencias como una dinámica que cobra sentido a partir de las complejas relaciones que se dan entre las imágenes de ciencia y conocimiento, la forma como se concibe la escuela y su papel en la sociedad y, las estrategias que se implementan en el aula para la construcción de explicaciones del mundo natural y social. En este escrito tales relaciones se describen en términos de referentes epistemológicos, pedagógicos y didácticos.

\section{REFERENTES EPISTEMOLÓGICOS}

El diseño de una propuesta de aula es un ejercicio académico que permite a los maestros abordar preguntas que tienen que ver con la naturaleza del conocimiento, sus procesos de producción y sus estrategias de legitimación social. Por lo anterior, se considera importante, por una parte, explicitar las relaciones y supuestos que permiten concebir la ciencia como una actividad cultural y por otra, describir algunas transformaciones que tienen lugar en la construcción de conocimiento en ciencias.

\section{La ciencia como construcción de representaciones del mundo natural y social}

El sentido y significado que asignamos al mundo está en relación con la representación que construimos de él a partir de nuestras vivencias. Esta afirmación no resulta novedosa, es uno de los principios constructivistas que ha animado las prácticas educativas de los últimos tiempos.

La manera a través de la cual el hombre da sentido y se relaciona consigo mismo y con su mundo natural y social, se enriquece de los múltiples espacios de significación que elabora en su devenir histórico individual y colectivo. Estos espacios de significación permiten la emergencia de representaciones en los sujetos, y son ellas las que movilizan diferentes formas de relación con el mundo, le permiten sobrevivir y adaptarse a medios hostiles, pero además le vinculan con la capacidad de construir conocimiento; esto es, conocer es representar y representar es conocer (Valencia y otros: 2000). Las representaciones son entendidas como construcciones discursivas con carácter ideológico que expresan relaciones de saber-poder que se dan en los contextos culturales donde circulan.

Desde este punto de vista, el interés por el papel de las representaciones en la construcción de conocimiento, no se centra en determinar si dichas representaciones son o no verdaderas o si se corresponden con un modo de representar dominante, sino en mostrar que es posible transformar las formas de relación que se establecen entre los sujetos y entre ellos y los saberes que circulan en la escuela, haciendo posible la coexistencia de múltiples formas de representar. En este sentido, las representaciones circulantes en la escuela configuran territorios de conflicto cultural que propician formas de relación equitativas y democráticas.

Ahora bien, si asumimos a la ciencia como una actividad de construcción de representaciones que coexisten con otras (religión, mito, arte, etc), se deriva la importancia de estudiar el papel que las dinámicas representacionales tienen en la construcción de 
nuevas realidades, es decir, en la construcción de narraciones locales de naturaleza, sociedad y hombre. Pensar la escuela como territorio de conflicto cultural, implica concebir los procesos que allí se dan como la expresión de relaciones de saber-poder, en donde las representaciones individuales se socializan, se alteran y coexisten con otras; esto es, hacen parte de un juego de explicitación, contrastación, enriquecimiento y transformación colectivo.

Finalmente, si reconocemos que conocer es representar y representar es conocer, se puede afirmar que en dicha actividad los sujetos comprometen las representaciones que han construido en sus diferentes espacios de actuación, sean estos cotidianos, televisivos, familiares, escolares, entre otros. Por tal razón, juegan un papel importante para el diseño de propuestas pedagógicas y didácticas alternativas para la escuela.

\section{De la experiencia básica a la complejización de las relaciones}

Dos aspectos que caracterizan la actividad del pensamiento contemporáneo son: la creciente complejización de los saberes y la constitución de relación sujeto-objeto como una unidad dialéctica de mutua constitución. Estos aspectos implican ciertas concesiones epistemológicas que cambian la forma como el sujeto se sitúa frente al mundo y las pretensiones desde las que orienta sus posibilidades de conocer. La concesión epistemológica sería entendida como una condición de negociación de significados, experiencias y concepciones del mundo que hace posible las transformaciones del pensamiento que le confiere un carácter gradual y fluido a las rupturas epistemológicas.

Una de estas concesiones tiene que ver con la relación sujeto-objeto. En el estudio de los fenómenos naturales y la indagación por las condiciones que han hecho de éstos objetos de conocimiento, quien indaga construye un espacio de relaciones que lo configuran como sujeto de conocimiento pero a la vez como objeto a conocer. En este sentido, la dialéctica sujeto-objeto desplaza las miradas reduccionistas y mecanicistas desde las cuales se consideran los fenómenos naturales como hechos aislados, por una dimensión renovada en la que adquieren sentido nociones como las de relación, emergencia y sistema.

Otra concesión se refiere al cuestionamiento de las aproximaciones inmediatistas y de las miradas simplistas que hacen del fenómeno natural algo obvio. Sólo a partir de la crítica de las estrategias espontáneas de conocer se puede configurar una mirada más detallada de los fenómenos y se avanza en la comprensión de las condiciones que hacen posible su emergencia.

En esta perspectiva, el análisis de las condiciones que permiten configurar explicaciones en diferentes espacios de enseñanza (educación básica, educación universitaria de pregrado y postgrado) ha permitido al equipo proponer la categoría problemas de conocimiento(PC). Ahora bien, en los PC los sujetos ponen en juego diferentes estrategias para construir explicaciones a situaciones del mundo natural y social; dichas estrategias se pueden describir en términos de: el cuestionamiento de la experiencia básica, la artificialización del mundo natural y la complejización de las relaciones. 


\section{El cuestionamiento de la experiencia básica}

La experiencia básica es la primera aproximación a los eventos del mundo natural. Ella permite sorprenderse con aquello que se reconoce novedoso, inquietarse con lo desconocido, maravillarse del universo que se habita. Este panorama en que se sumerge el pensamiento matiza la dificultad que comporta renunciar a las seguridades de una primera aproximación a los fenómenos y desde la que cobra sentido hablar de ellos como meras descripciones de una realidad dada e inmutable.

No obstante, en la perspectiva de comprender es necesario vencer las intuiciones primeras, el deslumbramiento y el entusiasmo por lo natural y vincularse al ejercicio del pensamiento; es decir, hacerle frente a las preguntas, dejarse enriquecer por el hecho construido y sancionado y movilizar el pensamiento renovándolo y rectificándolo incesantemente. Se entiende el cuestionamiento de la experiencia básica como la instancia que permite al sujeto adquirir un sentido renovado del fenómeno que le permite cuestionar las analogías primeras, enriquecer las metáforas, complejizar las descripciones y explicitar los sentidos. En este sentido, la búsqueda de principios, la verificación de leyes, la contrastación de teorías, la formulación de preguntas y la elaboración de supuestos constituyen actividades propias de esta instancia.

Se trata pues de cuestionar la morada que se construye desde los saberes cotidianos y desde la que se conciben seguridades primeras, para sumergirse en la incertidumbre de la pregunta, en la certeza de la construcción permanente, en la negociación de intereses y en la prospección de planes de trabajo, que movilizan al sujeto hacia formas de comprensión intencionada en la perspectiva de dar un orden al mundo que se experiencia.

\section{La artificialización del mundo natural}

Otra instancia del pensamiento es la condición desde la cual el sujeto se distancia de la experiencia básica en su interés por artificializar el mundo natural al imponerle condiciones e interrogarlo para obtener mayor información de él. En esta segunda instancia el sujeto se provee de elementos para la construcción de objetos de conocimiento y define estrategias para establecer variables, construir relaciones y derivar principios en su tránsito hacia la comprensión sistemática de los fenómenos. El fenómeno deja de estar en el plano de la seguridad de los saberes sancionados y empieza a ser definido como objeto de estudio y como espacio de posibilidades a la acción constructiva del pensamiento; es decir, el sujeto objetiviza el mundo.

En este sentido, el sujeto construye el fenómeno en la medida que da cuenta de las condiciones que permiten su existencia, de las variables que determinan sus cambios y de los procesos que lo constituyen. En otras palabras el sujeto se ve en la necesidad de proponer modelos, diseñar experimentos, simular situaciones, acuñar conceptos, anticipar eventos, elaborar generalizaciones y definir criterios metodológicos que enriquecen las explicaciones al fenómeno conocido. 


\section{La complejización de las relaciones}

Si bien la modelización es una instancia de comprensión, ella no agota la explicación del fenómeno. En el esfuerzo simplificador que le subyace a la modelización que tiene ver con el aislamiento de condiciones, la discriminación de variables y el proceder analítico se reconoce el carácter limitado y fragmentado con que usualmente son descritos los fenómenos naturales. En esta instancia de construcción del fenómeno se constatan los límites del pensamiento científico, el carácter limitado y suplementario de las teorías y la imposibilidad de atrapar el mundo en el discurso. En una renovada instancia la construcción del fenómeno es posible concebirla en su dimensión compleja lo cual demanda un cambio en las formas de mirar, de pensar y de hacer del sujeto que conoce. Se requiere un pensamiento capaz de dialogar, de negociar con lo real. Mientras el pensamiento simplificador desintegra, el nuevo pensamiento integra y conjuga los modos simplificadores de pensar; rechaza las consecuencias mutilantes, reduccionistas y unidimensionalizantes; aspira a un conocimiento multidimensional, que articule los dominios disciplinarios quebrados por el pensamiento disgregador; y se configure desde la tensión permanente entre la aspiración a un saber no acabado, no parcelado, no reduccionista y el reconocimiento de lo inacabado e incompleto de todo conocimiento.

La vinculación de los fenómenos al tiempo, la emergencia de nuevas relaciones, el reconocimiento de conexiones multicausales, el carácter complementario de las explicaciones, las codeterminaciones sujeto-objeto, las interrelaciones sistema-contexto, la recursividad de las concepciones y la naturaleza provisional de las teorías son expresiones de una manera compleja de ver el mundo.

\section{REFERENTES PEDAGÓGICOS}

Las dinámicas culturales del presente siglo en términos de la irrupción de las nuevas tecnologías de la información, la crisis de los grandes paradigmas del pensamiento occidental, la necesidad de generar alternativas de resistencia a los modelos de globalización cultural e ideológica, los desarrollos teóricos de campos disciplinares que han devenido en la superespecialización de los saberes, constituyen un territorio conceptual que plantea cuestionamientos a las formas de asumir los conocimientos -en particular el conocimiento científico- a las formas de su constitución y como tal a las prácticas sociales en donde estos se actualizan, es decir, las prácticas de su enseñanza.

El reconocimiento de estos nuevos escenarios para la acción pedagógica permite señalar algunos referentes para orientar prácticas alternativas de enseñanza de las ciencias que configuran la dinámica del aula como un espacio para: Por un lado, la constitución de sujetos sociales de conocimiento y con ello asistir a la emergencia de nuevas subjetividades y por otro, hacer significativa la actividad del aula desde la construcción de PC.

\section{La enseñanza de las ciencias y la emergencia de nuevas subjetividades}

Es posible pensar la escuela como territorio de expresión de los conflictos culturales, en donde maestros y estudiantes contrastan sus puntos de vista, sopesan el carácter de sus valoraciones y se embarcan en proyectos que transforman sus vínculos comunitarios locales; 
es decir, una escuela en donde los textos, los productos de la ciencia, los currículos, los programas, las valoraciones éticas, estéticas, lúdicas y las múltiples representaciones que la constituyen en territorio cultural, no son más que "insumos" para configurar dinámicas de renovación en las relaciones, para la emergencia de nuevos sentidos colectivos y para la construcción de nuevos órdenes educativos. Estamos hablando de una escuela que, al trascender su carácter de institución normalizadora que distribuye y adecua sujetos y saberes, se configura como territorio de transformación cultural.

De la misma manera, al estudiante de su condición de sujeto cognoscente al que hay que involucrar en procesos de enseñanza-aprendizaje que le permitan obtener logros y desarrollar competencias para instalarse en los saberes socialmente legitimados, es posible llegar a considerarlo como un sujeto social de conocimiento; es decir, un sujeto que se construye y reconstruye a partir de las múltiples relaciones que establece en su espacio cultural y que hace de la actividad de conocer una posibilidad para criticar permanentemente aquello que aparenta ser socialmente válido; un sujeto que elabora formas creativas de relacionarse consigo mismo, con los otros y con su entorno, y que es capaz de emocionarse con el conocimiento.

El maestro por su parte, deja de ser un distribuidor de saberes, para ser concebido como un posibilitador de dinámicas de transformación cultural y como un intelectual ideológicamente comprometido con nuevas maneras de entender los contextos en los que desarrolla su labor y en esta medida, aporta a la construcción de otros órdenes educativos. Para ello recupera sus intenciones, ejerce deliberadamente sus acciones y asume riesgos colectivos de propositividad, azar y transformación (Morin, 1996). Riesgos de propositividad, como apuestas que el maestro realiza con la certidumbre de su práctica y con el riesgo de convertirla en un acto creativo, desde donde es posible deslegitimar la experiencia como réplica del hacer, la confianza en la verdad de los textos y la normatividad que imponen los currículos, para dejarse sorprender con las preguntas de los estudiantes, los problemas de la comunidad, los temas que le apasionan y el placer de conocer. Riesgos de azar, como la expresión de una conciencia vigilante de sus propias acciones que hacen recurrente decidir cambios de itinerario, elegir caminos y desafiar la brújula que impone la rutina. Y riesgos de transformación que le permiten asumirse como protagonista de los nuevos sentidos que fortalecen su acción pedagógica, viviéndola, pero a la vez renovándola incesantemente.

Igualmente es necesario mirar críticamente las concepciones que muestran la ciencia como un "conjunto articulado de conocimientos dispuestos sistemáticamente dentro de un edificio cuya estructura firme comporta ya una manera de proceder, con estatutos acabados de verdad, con presupuestos inamovibles de objetividad, neutralidad y progreso, con técnicas infalibles de procedimiento, con razones para todo y criterios de autoridad que la sitúan por encima de la condición humana" (OROZCO: 1992), para construir una mirada más humana de la ciencia que permita asumirla como una representación social e históricamente condicionada por los entramados de la cultura.

Desde este contexto, la enseñanza de las ciencias se piensa como la puesta en acción de estrategias de transformación cultural que generan relaciones alternativas, con la información, con los otros, con la experiencia y con el entorno natural, en las que se actualiza 
el universo representacional de cada sujeto y es posible construir sentidos colectivos, diferentes formas de ser, hacer y pensar el mundo.

Asumir la enseñanza de las ciencias como una práctica cultural en donde se recuperan y recontextualizan los saberes, implica redimensionar el significado del aula. En efecto, ésta ya no puede verse como un espacio físico, sino como un sistema de relaciones sociales en donde las representaciones individuales se expresan, se alteran y coexisten con otras; esto es, un sistema de relaciones que permite a quienes participan expresarse, contrastarse, enriquecerse y transformarse colectivamente.

Desde este contexto, el bosque, la laguna, el caño o el parque, se configuran como espacios de relaciones en donde es posible pensar, soñar y construir el futuro en forma colectiva, actualizar el pensamiento y ponerlo en función para la construcción de múltiples realidades. Pensar el aula desde estas posibilidades permite potenciar en los sujetos la autoestima, el sentido crítico, la tolerancia por la diferencia y como tal, generar condiciones para la constitución de sujetos sociales de conocimiento.

En resumen, pensar el aula como un sistema complejo del que emergen nuevas formas de imaginar la vida escolar, es asignarle una condición ideológica, donde los sujetos que allí se relacionan contribuyen a construir nuevos sentidos culturales, inventar nuevas subjetividades y como tal generar posibilidades para existir creativamente.

\section{Los problemas de conocimiento y la enseñanza de las ciencias}

Los PC emergen como una categoría alternativa para comprender la construcción de conocimiento en ciencias. Desde esta categoría es posible pensar las ciencias y su enseñanza, en términos de actividad de la cultura, en donde lo que prima no es la reconstrucción de los corpus teórico-experimentales disciplinares, sino la generación de condiciones comunicativas y experienciales para la construcción de explicaciones del mundo físico. En la constitución de PC el sujeto transita por instancias tales como las descritas en los referentes epistemológicos desde las cuales paulatinamente configura nuevas maneras de relacionarse con los eventos del mundo natural y social.

Asumir la enseñanza de las ciencias desde la perspectiva de los PC, implica transcender la mirada del programa como el desarrollo de contenidos, hacia su comprensión como un proceso que se construye a partir de unas intenciones iniciales que se concretan $y$ transforman en el devenir de las prácticas escolares.

Tales intenciones se expresan en la construcción de espacios para el cuestionamiento de las representaciones de profesores y estudiantes, en el interés por formular problemas, en el apoyo al desarrollo de proyectos, en la necesidad de documentar preguntas, en fin, se expresan en la construcción de formas de abordar y formular soluciones alternativas a problemáticas del entorno natural y social. Así, preguntas como ¿Qué reacción tiene el sol sobre las plantas? ¿Cómo se desarrollan las orugas? ¿Cómo descontaminar un caño? ¿Qué contaminantes impiden el crecimiento de las plantas?, que en otros contextos pueden resultar obvias, se constituyen en experiencias que desencadenan procesos alternativos para la enseñanza de las ciencias y llevan al desarrollo de procesos de conocimiento. 
El problema de conocimiento se enriquece con la diversidad y heterogeneidad en las formas de explicar, cuestionar, abordar la investigación, y presentar los resultados, que son dados por las distintas vivencias de conocimiento tanto en el ámbito escolar como no escolar. Toda opinión, desde cualquier enfoque resulta pertinente en un proceso de construcción de un PC, ellas aportan los puntos de confluencia y divergencia que hacen más rico el proceso y que obligan a afinar la argumentación, que es requisito indispensable para la creación colectiva de explicaciones.

En esta perspectiva, los PC configuran la actividad que vivencian un grupo de sujetos que comparten preguntas alrededor de una situación particular, donde hacen una continua construcción de criterios o elementos que permitan ir validando los desarrollos del grupo y a su vez crean un lenguaje propio que se expresa no sólo en el lenguaje articulado, sino también en el gráfico. En últimas, un PC, sólo se puede pensar desde la idea de construcción continua de preguntas, acciones, argumentos, diseños experimentales, entre otros.

\section{REFERENTES DIDÁCTICOS}

En la definición y el diseño de propuestas para la enseñanza es importante tomar en cuenta: el papel de los intereses de los estudiantes, las motivaciones que permiten definir las temáticas y problemas a trabajar; el tipo de relaciones con los textos, el uso de material audiovisual, las salidas pedagógicas y las socializaciones, entre otros. La reflexión sobre estos aspectos permite definir algunos referentes didácticos que están en relación con los referentes epistemológicos y pedagógicos desde los que se orientan prácticas alternativas para la enseñanza. La didáctica no se asume como una disciplina autónoma e independiente para orientar los procesos del aula, ni como un saber instrumental subordinado a la pedagogía.

Reconocer la complejidad y particularidad en que se desenvuelve la actividad del aula permite delimitar algunos criterios de actuación y unas formas de trabajo que concretan procesos implicados en la constitución de PC.

De estos aspectos se deriva que ningún proceso puede ser igual a otro, esto amerita realizar lecturas de las vivencias de cada grupo cuando se desarrolla una propuesta, de tal manera que se pueda dar cuenta que el carácter dinámico y transformador de los procesos de construcción de conocimiento está mediado por el tipo de relaciones que se configuran en el aula.

\section{Criterios de actuación}

Los PC expresan la vivencia del aula y se involucran en el diario vivir, en actividades como: comunicar las construcciones gráficas o textuales, salidas exploratorias del entorno, configuración de grupos según sus intereses y preocupaciones, búsqueda de preguntas, diseño de planes de trabajo, discusión de las elaboraciones, documentación con otras fuentes de conocimiento, participación en talleres, elaboración de textos y cartillas y sobre todo desde las transformaciones que sufren las preguntas, las representaciones y las propuestas experimentales. En este sentido, el PC se define desde la significación y 
resignificación que se le da a una situación o fenómeno de estudio, esto es, es la actividad misma de conocer. De esta manera, no se pueden definir instrucciones que permitan configurar PC en el aula, se considera más adecuado esbozar algunos criterios de actuación entendidos como estrategias que permiten al maestro aportar elementos para su constitución.

\section{Situaciones desencadenantes}

El enfoque por PC relativiza la idea de una comprensión paulatina de los saberes disciplinares como única función del aula y cuestiona la tendencia a separar espacios teóricos donde se definen principios, leyes y teorías de espacios prácticos en los que los estudiantes comprueban en situaciones experimentales dichos enunciados definidos como verdades universales, atemporales y descontextualizadas. En contraste, la actividad del aula se configura colectivamente y en ella el maestro juega un papel fundamental en el reconocimiento de los intereses de los estudiantes, en la definición intencionada de los propósitos y en la orientación de los procesos.

En los PC la actividad del aula se alimenta y activa permanentemente por las preguntas de los participantes, de manera que cada socialización entendida como un alto en el camino para hacer balance de la actividad se constituye en un nuevo comienzo que configura opciones de salidas, genera interrogantes, organiza situaciones experimentales, afina las descripciones, documenta los argumentos, entre otros aspectos que enriquecen las posibilidades explicativas a nivel individual y colectivo. Esto permite, que cuando el maestro describa la actividad del aula pueda reconocer en su devenir una imagen de espiral en la que cada vez se compeljiza más la situación o fenómeno de estudio y se accede a un nuevo campo de problemas.

Las situaciones desencadenantes solo pueden ser descritas cuando el maestro reflexiona sobre la actividad del aula, no se definen a priori, no se prescriben, por el contrario emergen en la confluencia de preguntas, en la contrastación de hipótesis, en la delimitación colectiva de intereses, en el comentario improvisado, entre otros.

Las situaciones desencadenantes se constituyen en espacios para la problematización de situaciones o eventos del mundo cotidiano que permiten a un grupo de individuos orientar y delimitar sus intereses en la perspectiva de construir conocimiento.

\section{Relaciones Alternativas}

Organizar la actividad del aula en la perspectiva de constituir PC permite alterar las relaciones que los sujetos establecen en su devenir; estas relaciones dejan de ser unidireccionales y atemporales y comienzan a estar permeadas por el contexto donde se constituyen las nuevas subjetividades del maestro y el estudiante.

Las relaciones alternativas con la información, se expresan en el cambio de concepción que los estudiantes tienen de ella; esto es, la información deja de ser sinónimo de conocimiento y 
empieza a ser concebida como referente que documenta las preguntas, amplía las experiencias y enriquece las explicaciones.

Las relaciones alternativas con los otros redimensionan el papel del compañero y del profesor. Del primero, en el sentido en que se comienza a ver como alguien del cual se aprende y del profesor como alguien que reconoce que "no lo sabe todo". Una relación que permite concebir al otro como compañero de viaje en la actividad de conocer, con el cual puede intercambiar sus rutas, pensar colectivamente los itinerarios, ubicar las condiciones propicias para orientar la experiencia; es decir, un otro a través del cual se construye y reconstruye permanentemente.

Las relaciones alternativas con el entorno natural en las que éste deja de ser visto como recipiente que contiene cosas y en el que ocurren eventos y empieza a ser comprendido como sistema de relaciones del cual somos emergencia. Una relación en donde los problemas del entorno se asumen como propios, pues se pertenece al espacio desde donde se configuran.

Y por último, relaciones alternativas con la experiencia que fortalecen la inquietud por conocer, resuelven la necesidad de contrastar, experienciar aquello que nos parece incomprensible, vivenciar nuevas formas de relación con las preguntas que nos asechan, imaginar mundos donde nuestras preguntas configuran sus sentidos y artificializar el universo de nuestra indagación.

\section{Ambientes comunicativos}

Reconocer que el sujeto es y se configura a través de la palabra hace necesario propiciar actividades en el aula que permitan a los individuos hablar, escribir y escuchar como elementos indispensables para el intercambio de opiniones, puntos de vista, pareceres, deseos, inquietudes. Para esta propuesta, el otro es más que un individuo o un grupo de ellos, es aquel que se revela desde su diferencia o heterogeneidad, por medio de sus escritos, aportes en las discusiones, participación en la elaboración de informes y en el reconocimiento y respeto por la producción de sus pares.

La expresión de diversas formas de ver, escuchar, relacionar y comprender permite al estudiante desinhibirse, reconocerse como ser creativo, valorar sus propias producciones e iniciar procesos de construcción de conocimiento de manera intersubjetiva. Lo anterior permite validar la idea de la clase de ciencias como espacio donde el estudiante formula preguntas, delimita problemas, expresa inquietudes diseña alternativas de trabajo para el año lectivo. Elementos que a nuestro juicio, aportan en el reconocimiento, por parte de estudiantes y maestros, de la ciencia como una actividad de la cultura.

\section{Formas de trabajo}

Configurar un ambiente de trabajo en el aula, en donde las responsabilidades individuales, los intereses, las opiniones y el deseo de saber se conjuguen en una búsqueda común, propicia el diseño y el desarrollo de proyectos. En esta nueva organización se generan las condiciones para la construcción de explicaciones, el desarrollo de argumentaciones y la 
solución de problemas significativos. Esto es posible desde actividades como: salidas pedagógicas, desarrollo de planes de trabajo, realización de talleres, elaboración de materiales de divulgación.

Las salidas pedagógicas constituyen actividades a través de las cuales el aula se abre; así es como el parque, el caño, el bosque, la visita dirigida, la exploración del entorno institucional son pretextos para el reconocimiento de los intereses, la definición de preguntas, la construcción de problemas y el desencadenamiento de procesos explicativos.

Los planes de trabajo permiten organizar y proyectar colectivamente lo que se va a hacer, los procedimientos que se van a seguir para ello y los recursos implicados. Los planes a su vez recuperan las intenciones de los estudiantes y maestros en la construcción de rutas de viaje, la definición de estrategias metodológicas y experimentales, la consecución de fuentes bibliográficas o testimoniales, entre otras. En ellos se expresan las experiencias escolares, cotidianas, sociales desde las cuales el sujeto vivencia la actividad de conocer.

Los talleres son actividades diseñadas por el maestro a partir del análisis permanente de los sucesos del aula; con ellos se pretende precisar contenidos, desarrollar habilidades metodológicas, articular hallazgos y promover la discusión entre grupos.

La elaboración de materiales de divulgación constituye un ejercicio escritural que enfrenta a los estudiantes con nuevas formas de elaborar explicaciones, organizar sus ideas y comunicar sus vivencias y aprendizajes. Así, por ejemplo, deben decidir lo que quieren comunicar, diseñar formatos, ilustrar, elegir títulos o seleccionar gráficos, este proceso lleva al estudiante a hacer conciencia de las estrategias más adecuadas para comunicar a los otros sus hallazgos y como tal, asumir el conocimiento de una forma intencionada. La dinámica se caracteriza por la elaboración individual y colectiva de textos y bocetos. En esta tensión individuo-grupo, los participantes vivencian que las historias individuales entretejen historias colectivas.

\section{BIBLIOGRAFÍA}

ESCOBAR Arturo. 1996. La invención del Tercer Mundo. Construcción y deconstrucción del desarrollo. Editorial Norma. Bogotá.

FOUCAULT, Michel. 1970. El orden del discurso. Cuadernos Marginales. México

FOUREZ, G. 1994. Alfabetización Científica y Tecnológica. Colihue. Buenos Aires.

WATZLAWICK Paul y otros. 1981.La realidad Inventada. Gedisa Editorial. Barcelona.

TORRES J. 2000. Globalización e interdisciplinariedad: el curriculum integrado. Morata. Madrid.

LASZLO Ervin. 1990. La gran bifurcación. Editorial Gedisa. Barcelona.

MORIN, E., 1983. El método. La vida de la vida. Cátedra. Madrid.

MORIN, Edgar; 1995. El método: la naturaleza de la naturaleza. Alianza. Barcelona.

OROZCO C. Juan Carlos. La ciencia se imagina en el espíritu científico. En: Rev. de investigaciones. Vol V. No. 1. Uni. Nariño 1991-1992. pp. 113.

PRIGOGINE Ilya. 1997. El fin de las certidumbres. Taurus. Madrid.

SEGURA D. y otros. 1995.Vivencias de conocimiento y cambio cultural. El Fuego Azul. Bogotá. 
VALENCIA V., S., MÉNDEZ N., O., JIMÉNEZ G., G., GARZÓN O., J. P. De la contemplación a la comprensión de los seres vivos. En: Campo Abierto Revista de Educación. v.20, p.29 46,2000

VIRILIO Paul. 1997. El cibermundo, la política de lo peor. Ediciones Cátedra. Madrid. 